\title{
Validação de cartilha para promoção do conforto de familiares com parentes hospitalizados
}

\author{
Validation of a booklet designed to promote comfort of relatives of hospitalized patients
}

Jaqueline Sena Muniz ${ }^{1}$, Kátia Santana Freitas ${ }^{1}$, Joselice Almeida Góis ${ }^{1}$, Adriana Braitt Lima ${ }^{1}$, Elaine Guedes Fontoura $^{1}$, Marluce Alves Nunes Oliveira ${ }^{1}$

Objetivo: validar o conteúdo de cartilha educativa para promoção do conforto de familiares com parentes hospitalizados. Métodos: estudo metodológico, desenvolvido em Unidade de Terapia Intensiva, em três etapas: construção de cartilha educativa, validação por especialistas e análise semântica. Para análise do grau de relevância e pertinência do conteúdo e imagens, empregou-se o Índice de Validade de Conteúdo e adotaram-se os valores $\geq 0,80$. Resultados: a cartilha obteve avaliação satisfatória em relação aos critérios avaliados, obtendo Índice de Validade de Conteúdo geral de 0,87. Os itens que não alcançaram índice de validade de conteúdo adotado foram reformulados. Na avaliação realizada pelos juízes, evidenciou-se adequação do conteúdo às principais necessidades de informação. Conclusão: o conteúdo da cartilha educativa apresentou índices de validade adequados, constituindo-se subsídio para esclarecer dúvidas de familiares, sendo, portanto, ferramenta para educação em saúde.

Descritores: Estudos de Validação; Materiais de Ensino; Unidade de Terapia Intensiva; Enfermagem Familiar.

Objective: to validate the content of an educational booklet to promote comfort of relatives of hospitalized patients. Methods: methodological study developed in an Intensive Care Unit carried out in three stages: construction of the educational booklet, expert validation, and semantic analysis. The Content Validity Index was used to analyze the degree of relevance and pertinence of the content and the images; values $\geq 0.80$ were adopted. Results: the booklet obtained a satisfactory evaluation in to the evaluated criteria, with an overall Content Validity Index of 0.87 . Items that did not reach the content validity index adopted were reformulated. The evaluation made by the judges evidenced that the content was adequate to the main information needs. Conclusion: the content of the educational booklet presented adequate validity indices, thus representing an aid to clarify doubts of relatives, and being, therefore, a tool for health education.

Descriptors: Validation Studies; Teaching Materials; Intensive Care Units; Family Nursing.

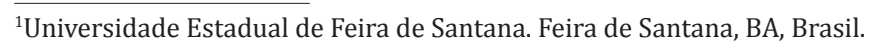




\section{Introdução}

0 internamento de uma pessoa em Unidade de Terapia Intensiva, geralmente, ocorre de forma repentina, contribuindo para desajustamento familiar, à medida que diversos desconfortos são vividos. As descomodidades de familiares neste contexto têm sido entendidas como alterações, perturbações e dificuldades de natureza física, psíquica e social, decorrentes da interação do familiar com a hospitalização ${ }^{(1)}$. Dentre estas, os sentimentos de angústia, medo pelo desconhecimento da real condição clínica do parente, impotência diante do tratamento e mudança para ambiente cujas normas e rotinas são estranhas. Tais desconfortos atingem de forma direta o núcleo familiar, ao provocar alterações emocionais e, inevitavelmente, interrupção na vida diária de $\operatorname{membros}^{(2)}$.

Ademais, existem outros fatores que colaboram e potencializam os desconfortos ou confortos desses familiares, como possuir informação sobre a evolução clínica do parente, com indicadores consistentes de orientação clara e sincera que ajudam a minimizar dúvidas, o medo da incerteza do destino do ente ou, até mesmo, a ausência de controle da situação vivida, configurando-se como elemento essencial para o acolhimento ${ }^{(2-3)}$.

A carência de orientações pode acentuar o desequilíbrio emocional dessas famílias. Essa necessidade pode ser atendida, através da conversa com profissionais da equipe de saúde durante as visitas, ao presenciar e interagir com o parente, bem como por meio de impressos educativos ${ }^{(4)}$. A informação escrita por intermédio de materiais, como folhetos e cartilhas, tem sido utilizada para melhorar o conforto de famílias em situação de hospitalização.

0 material educativo impresso tem sido ferramenta útil, por ser considerada tecnologia educativa aplicada na educação em saúde, pode melhorar o conhecimento, a satisfação e a aderência, não somente ao tratamento, como também ao autocuidado de pacientes em diversas situações de adoecimento ${ }^{(5-6)}$. Neste sentido, a cartilha educativa é uma das estraté- gias utilizadas para promoção do conforto, por meio da entrega de informações e orientações escritas a familiares no contexto hospitalar. Sabe-se que a família aprecia as orientações escritas e estas funcionam como recursos prontamente disponíveis, pois estão ao alcance desta, podendo consultá-la, quando necessário, o que favorece a organização do grupo familiar e a ativação de mecanismos de enfrentamento da situação $0^{(7)}$.

No entanto, para que essas informações obtenham a eficácia esperada, é necessário processo de validação, no qual visa avaliar os pontos imprescindíveis na compreensão do leitor quanto à realidade vivenciada, a adequação do conteúdo e da linguagem ${ }^{(8)}$.

A importância da informação para o conforto da família, seja verbal ou escrita, tem sido descrita na literatura ${ }^{(9)}$, no entanto, o número de publicações sobre o processo de elaboração e disponibilização de cartilhas educativas para orientação de familiares de pacientes internados em terapia intensiva é escasso, dificultando ainda mais o processo de desenvolvimento sistemático destas. A carência de estudos que abordem as práticas adotadas na elaboração de materiais para familiares no contexto da hospitalização constituiu elemento motivador. A realização de buscas nas bases de dados: Literatura Latino-Americana e do Caribe em Ciências da Saúde, Sistema On-line de Busca e Análise de Literatura Médica e Scientific Electronic Library Online, empregando primeiro as palavras-chave: cartilha, família e cuidados intensivos, sem estabelecer limite de tempo, não revelou artigo relacionado diretamente à temática, evidenciando lacuna na produção de estudos para família, na perspectiva de conforto.

O conteúdo da cartilha proposta pode ser válido para minimizar os desconfortos vivenciados por familiares, como melhorar o bem-estar físico, psicológico, espiritual e, consequentemente, ajudá-los no enfrentamento e na tomada de decisões, de modo que fortalecidos consigam enfrentar a experiência de ter um familiar na terapia intensiva. Assim, tendo por meta a promoção de conforto, questionou-se: a cartilha 
elaborada para promoção do conforto de famílias na terapia intensiva possui índices de validade de conteúdo adequados? Logo, objetivou-se elaborar e validar o conteúdo de cartilha educativa para promoção do conforto de familiares com parentes hospitalizados.

\section{Métodos}

Estudo metodológico, realizado de março a julho de 2017. 0 cenário do estudo foi hospital público de grande porte, no interior da Bahia, Brasil, trata-se de instituição governamental que possui duas Unidades de Terapia Intensiva adulto que totalizam a quantidade de 18 leitos. Os participantes do estudo foram oito especialistas, com experiência na temática, e oito familiares que vivenciaram a experiência de possuir um familiar hospitalizado na referida unidade hospitalar.

Por se tratar de manual informativo, os procedimentos teóricos para desenvolvimento do material incluíram desde a explicitação da teoria sobre o objeto em estudo, até a elaboração da cartilha informativa para família. A primeira etapa consistiu na construção das temáticas da cartilha educativa, e para tanto foi realizado levantamento bibliográfico em artigos científicos e bases de dados, sem estabelecer intervalo de tempo, sobre as necessidades, sentimentos, conforto e desconforto de famílias em situação de hospitalização, objetivando construir cartilha baseada em evidências científicas. Ao total, foram selecionados 21 artigos que abordavam as principais necessidades de informações de familiares em situação de hospitalização. Após seleção e leitura dos materiais, realizou-se a categorização das informações identificadas como importantes para famílias, as quais deram origem as temáticas apresentadas na cartilha educativa. Posteriormente, elaborouse a estrutura interna da cartilha, que consistiu na junção das partes textuais com as ilustrações, tendo como critério de seleção que as imagens se aproximassem da realidade encontrada por familiares no hospital. Em seguida, procedeu-se à formatação do material, ao qual resultou no primeiro protótipo da cartilha que foi encaminhando para impressão.

A cartilha educativa foi intitulada: "O que o familiar precisa saber quando tem um ente na Terapia Intensiva?" Possui 14 folhas, sendo composta por elementos pré-textuais (capa e apresentação), textuais (seis temáticas, desde a finalidade da Unidade de Terapia Intensiva até orientações sobre conforto para os familiares) e pós-textuais (referências).

Para tornar as informações compreensíveis ao público-alvo, adotou-se linguagem simples e de fácil entendimento. Os temas abordados foram: 0 que é a Unidade de Terapia Intensiva? 0 que é a sala de espera? Quem são os profissionais de saúde que trabalham nesse setor? Recursos utilizados para o tratamento? Visita hospitalar, Finalidade do boletim médico e Como se cuidar durante a internação do seu parente na Unidade de Terapia Intensiva?

A segunda etapa consistiu na análise da cartilha por especialistas. A cartilha piloto foi submetida à análise de especialistas para julgarem e opinarem sobre a correspondência dos itens selecionados ao objeto de interesse central, no caso deste estudo, as informações aos familiares de pessoas em estado crítico de saúde. A cartilha foi avaliada por comitê de oito juízes especialistas, tendo como critério de inclusão: ser pesquisador ou profissional de saúde, com experiência em terapia intensiva e/ou com famílias em situação de hospitalização, além de atuar/atuando no período diurno, já que neste ocorria a interação com os familiares.

Esses especialistas foram contatados através de carta convite, na qual se explicou sobre objetivo da pesquisa e importância da avaliação, sendo acompanhada pela declaração de aceite e formulário específico, contendo pelos menos três itens para avaliar cada temática da cartilha.

Os juízes, inicialmente, avaliaram o instrumento por temáticas, verificaram se cada conteúdo foi adequadamente abordado, se todas as dimensões que representavam o objeto de interesse foram incluídas. Posteriormente, as temáticas da cartilha foram analisadas em itens, quanto a possuir clareza e pertinên- 
cia, considerando escala tipo Likert de quatro pontos: 1-não, 2-pouco, 3-parcialmente e 4-totalmente, sendo solicitado que ao marcar as opções 1 e 2 , apresentassem a justificativa em espaço reservado para avaliação descritiva dos juízes. Em relação à clareza, verificouse a redação e a compreensibilidade dos conteúdos. Na análise da pertinência, avaliaram-se se os conteúdos de cada tema refletiam os conceitos envolvidos, se eram relevantes e suficientes para atingir o objetivo proposto. Analisaram-se, ainda, a adequação das imagens à realidade vivenciada por familiares.

A avaliação dos juízes se realizou por meio de formulário de pesquisa on-line, denominado de Google Forms, que compõem um dos leques de aplicativos do Google Drive. Após análise dos juízes, a cartilha foi reavaliada e as sugestões para modificações necessárias ao aperfeiçoamento foram realizadas e mantidos na versão piloto II, a fim de ser avaliado pelos próprios familiares.

A terceira etapa consistiu na análise semântica pelo público-alvo. Após análise dos especialistas, a segunda versão da cartilha piloto foi submetida à avaliação da análise semântica, a fim de verificar se o conteúdo da cartilha estava compreensível ao público que se destina. Nesta etapa, foram convidados, na sala de espera, oito familiares com parentes em tempo de internação igual ou superior a três dias, pois se estimou que nesse período o familiar reconhecia as principais necessidades de informações. Após aceite para participação, assinaram o Termo de Consentimento Livre e Esclarecido. A avaliação ocorreu de forma individual, utilizando instrumento impresso semelhante ao utilizado pelos especialistas, entretanto com foco na clareza das informações.

Para medir a proporção ou porcentagem de juízes que concordavam sobre aspectos da cartilha, calculou-se o Índice de Validade de Conteúdo (IVC), por meio da soma de concordância dos itens que receberam a pontuação 3 ou 4 pelos especialistas, dividido pelo número total de respostas. Para o cálculo do IVC global das temáticas, realizou-se a média aritmética dos IVC individuais. O IVC considerado adequado foi $\geq 0,80$. Itens que tiveram IVC inferior a esse valor foram revisados ${ }^{(10)}$.

Este estudo foi aprovado pelo comitê de ética em pesquisa da Universidade Estadual de Feira de Santana, o qual foi aprovado conforme parecer 1.758.708/16.

\section{Resultados}

O processo de validação de conteúdo foi iniciado após análise realizada por um comitê de oito especialistas, sendo seis profissionais de saúde que atuavam no hospital no período diurno (um médico, três enfermeiros, um psicólogo, um assistente social) e dois pesquisadores na área que foram selecionados por meios de publicações. Possuíam mais de cinco anos de experiência no objeto de interesse central, a maioria do sexo feminino, com idade acima de 30 anos.

A avaliação da cartilha ocorreu por temáticas, verificando se cada conteúdo foi adequadamente contemplado. Posteriormente, a cartilha foi analisada segundo os objetivos, a estrutura e apresentação.

Na avaliação geral da cartilha, apenas dois itens tiveram IVC individual menor que o estabelecido. Em relação ao domínio estrutura e apresentação, os menores escores foram referentes aos aspectos gramaticais $(0,62)$ e à adequação das imagens à realidade vivenciada $(0,75)$, sendo recorrigidos conforme as sugestões dos avaliadores.

Os itens referentes aos objetivos obtiveram a pontuação máxima (IVC individual e global = 1), mostrando que realmente a cartilha elaborada abordava as informações que mais provocavam dúvidas e curiosidades entre os familiares.

Em estrutura e apresentação, os juízes consideraram que o instrumento informativo era apropriado para os familiares que tinham parente na terapia intensiva (IVC=1), as informações estavam cientificamente claras e possuíam sequência lógica (IVC=1), 
a linguagem estava apropriada ao nível de escolaridade do público-alvo (IVC=0,87), a formatação da capa, apresentação estavam coerentes (IVC=0,87), o tamanho de letras e quantidades de páginas estavam adequadas (IVC=1). A avaliação dos especialistas em relação à estrutura e apresentação apontou recomendações, principalmente no item referente à concordância e ortografia dos textos (IVC $=0,62$ ), assim todos os itens sinalizados foram revistos e corrigidos.

$\mathrm{Na}$ avalição por temáticas, no tópico $\mathrm{O}$ que é a Unidade de Terapia Intensiva?, e o que é a Sala de espera?, os juízes avaliaram a forma que os temas foram explicados e a acessibilidade ao público-alvo, a relevância das informações e adequação das imagens à realidade vivenciada. 0 item que obteve menor escore foi referente à qualidade das imagens, obtendo IVC individual 0,75 e como recomendação: reavaliar a nitidez das imagens, sugestão acatada e incorporada na cartilha (Figura 1). No geral, as temáticas tiveram boa avaliação, alcançando IVC global 0,87.

O tema sobre os profissionais de saúde que trabalham na terapia intensiva obteve o menor IVC individual e global $(0,75)$. Os juízes consideraram que era necessário adequar algumas informações para melhor compressão dos familiares, e como sugestões, incluir outros profissionais de saúde, além dos contemplados na cartilha, dentre estes: assistente social, fonoaudió logo e dentista. $\mathrm{O}$ item de menor IVC individual foi referente às competências dos profissionais de saúde, tendo como escore 0,50, cujas principais recomendações foram: rever as competências da equipe de enfermagem e do médico intensivista. Em relação à adequação das imagens (IVC= 0,75$)$, sugeriu-se trazer imagens de profissionais brasileiros em atuação. Nessa temática, a clareza do item obteve maior avaliação, alcançando IVC individual 0,87.

A temática referente aos recursos utilizados no tratamento do familiar internado, também, necessitou ser revisada, pois obteve IVC global o escore de 0,74 e a clareza da linguagem adotada para explicação dos mesmos $(0,75)$. 0 item sobre os dispositivos e/ou equipamentos que mais despertavam dúvidas nos familiares obteve o menor escore $(0,62)$, sendo ajustado conforme as principais sugestões dos especialistas em relação à inclusão de alguns dispositivos: o ventilador mecânico junto ao respirador, o cateter de oxigênio e a sonda nasogástrica. Também, explicou-se sobre a finalidade da traqueostomia e do colchão "caixa de ovo", termos considerados frequentes no cotidiano hospitalar e importantes para o entendimento dos familiares, uma vez que o item referente à qualidade das imagens obteve o maior IVC individual $(0,87)$.

\begin{tabular}{|l|l|l|}
\hline Temas/Itens & \multicolumn{1}{|c|}{ Sugestões dos juízes especialistas } & IVC* \\
\hline $\begin{array}{l}\text { As ilustrações estão adequadas ao } \\
\text { conteúdo? }\end{array}$ & $\begin{array}{l}\text { Está pouco nítida, impedindo que os familiares percebam aspectos importantes no } \\
\text { ambiente; rever a qualidade das imagens disponibilizadas na cartilha, pois estão sem foco. }\end{array}$ & 0,75 \\
\hline $\begin{array}{l}\text { A equipe de saúde da unidade de tera- } \\
\text { pia intensiva apresentada no material } \\
\text { está coerente com a realidade? }\end{array}$ & $\begin{array}{l}\text { Mesmo que o (a) assistente social não esteja lotado (a) na unidade os familiares podem } \\
\text { contar com o apoio da Equipe do Serviço Social da Emergência; incluir o profissional }\end{array}$ & 0,75 \\
\hline $\begin{array}{l}\text { As definições demonstram o papel de e fonoaudiólogo, na especificação dos profissionais. } \\
\text { cada profissional na prestação do cui- } \\
\text { dado? }\end{array}$ & $\begin{array}{l}\text { Acrescentar em: funções do enfermeiro para realização de cuidados mais complexos; } \\
\text { enfermagem: preparo de soluções, oferta de dieta à pessoa internada, quando dieta via } \\
\text { oral. }\end{array}$ & 0,50 \\
\hline As ilustrações estão adequadas? & Trazer imagens de profissionais brasileiros e em atuação. & 0,75 \\
\hline
\end{tabular}

Figura 1 - Especificação dos itens do instrumento informativo que obtiveram IVC $<0,80$ quanto às temáticas 
Os temas que abordaram visita hospitalar, boletim médico e como se cuidar durante o internamento do parente atingiram o IVC individual e global elevados, portanto não precisaram ser reformulados. Os juízes consideraram que estavam claros e a abordagem estava intimamente relacionada às necessidades dos familiares. A seguir, na Tabela 1, estão os resultados da avaliação dos familiares participantes.

Após realizar as alterações sugeridas pelos juízes, a cartilha foi submetida à avaliação pelo público-alvo. Esta etapa contou com a participação de oito familiares, em maioria mulheres, entre 20 e 30 anos, filhas, com tempo de internação entre sete e 10 dias. Na avaliação do público-alvo, observou-se o Índice de
Validade de Conteúdo, referente à análise semântica, segundo as temáticas (Tabela 1 ).

Os temas o que é Unidade de terapia intensiva/ sala de espera, Recursos utilizados para o tratamento, Boletim médico e Como se cuidar durante o internamento alcançaram os maiores IVC global e individual. A temática "Quem são os profissionais que trabalham na terapia intensiva?" alcançou IVC global 0,8 , porém um item avaliativo relacionado ao conhecimento dos familiares acerca dos profissionais de saúde, apresentados na cartilha, obteve IVC $=0,20$, justificado pelo desconhecimento da família sobre a diversidade de profissões que atuam na Unidade de Terapia Intensiva.

Tabela 1 - Análise das temáticas da cartilha informativa por familiares

\begin{tabular}{|c|c|c|}
\hline Temáticas & IVC* & IVC global \\
\hline \multicolumn{3}{|l|}{ O que é a Unidade de terapia intensiva? E o que é a Sala de espera? } \\
\hline Os temas foram explicados de forma simples e de fácil entendimento? & 1,0 & \multirow{3}{*}{0,91} \\
\hline As informações esclarecem suas principais dúvidas? & 0,87 & \\
\hline As imagens retratam a realidade encontrada no hospital? & 0,87 & \\
\hline \multicolumn{3}{|l|}{ Quem são os profissionais de saúde que trabalham na Unidade de Terapia Intensiva? } \\
\hline Você já conhecia os profissionais de saúde citados na cartilha? & 0,20 & \multirow{4}{*}{0,80} \\
\hline As definições sobre o papel de cada profissional são de fácil entendimento? & 1,0 & \\
\hline O tema foi explicado de forma simples? & 1,0 & \\
\hline As imagens estão adequadas ao tema? & 1,0 & \\
\hline \multicolumn{3}{|l|}{ Recursos utilizados para tratamento na Unidade de Terapia Intensiva } \\
\hline $\begin{array}{l}\text { Os dispositivos e/ou equipamentos apresentados na cartilha eram os que mais lhe provocavam dúvidas ou } \\
\text { curiosidades? }\end{array}$ & 0,87 & \multirow[t]{2}{*}{0,91} \\
\hline A explicação sobre a função de cada dispositivo e/ou equipamentos foi realizada de forma clara? & 0,87 & \\
\hline As imagens são semelhantes às encontradas na Unidade de Terapia Intensiva? & 1,0 & \\
\hline
\end{tabular}

Tabela 2 - Análise das temáticas da cartilha informativa por familiares

\begin{tabular}{|c|c|c|}
\hline Temáticas & IVC* & IVC global \\
\hline \multicolumn{3}{|l|}{ Visita hospitalar } \\
\hline O tema esclarece suas principais dúvidas? & 0,87 & \multirow{3}{*}{0,83} \\
\hline As informações são suficientes? & 0,75 & \\
\hline As dicas citadas são importantes e de fácil entendimento? & 0,87 & \\
\hline \multicolumn{3}{|l|}{ Boletim médico: hora da informação } \\
\hline O tema esclarece suas principais dúvidas? & 0.87 & \multirow{3}{*}{0,91} \\
\hline As informações estão escritas de forma clara? & 0.87 & \\
\hline As dicas do que perguntar ao profissional médico são importantes? & 1,0 & \\
\hline \multicolumn{3}{|c|}{ Como se cuidar durante o internamento do parente na Unidade de Terapia Intensiva? } \\
\hline O tema está escrito de forma simples? & 1,0 & \multirow{2}{*}{0,96} \\
\hline As informações são suficientes? & 1,0 & \\
\hline As dicas são importantes para lhe ajudar a enfrentar esse momento? & 0,87 & \\
\hline
\end{tabular}


Além disso, verifica-se na Tabela 2, que na temática Visita hospitalar, a família apontou como insuficiente a quantidade de informações (IVC $=0,75$ ). Após avaliação pelos familiares participantes, constatou-se que nenhum item precisou ser retirado. Os familiares consideraram que os temas foram redigidos de forma clara e que as informações abordadas eram importantes para que enfrentem essa experiência mais fortalecidos.

\section{Discussão}

A impossibilidade de avaliar a efetividade da cartilha na prática clínica para redução de níveis de ansiedade e desconforto foi a principal limitação deste estudo, despontando como perspectiva futura de pesquisa.

As tecnologias educativas como estratégia para atender às necessidades de informações têm sido reconhecidas como importante ferramenta para o acolhimento e a promoção de conforto de pessoas em situação de adoecimento agudo e respectivos familiares. Essas ferramentas conseguem instrumentalizar a família em determinados aspectos da experiência que não são conversados no cotidiano da hospitalização com profissionais de saúde, favorecendo o enfrentamento da família e, até mesmo, a tomada de decisão relacionada ao parente em adoecimento ${ }^{(11)}$.

Em estudo sobre a importância da comunicação com familiares internados na terapia intensiva, constatou-se que o contato com os familiares era restrito ao horário de visita e, em geral, a equipe não tinha alcançado às necessidades informativas e emocionais da família, podendo transformar a experiência de internação em processo negativo e frustrante ${ }^{(12)}$. As ações desempenhadas, antecipadamente, por toda equipe multiprofissional pode contemplar as dúvidas mais frequentes e questionamentos que ainda não surgiram, produzindo a percepção de humanização do ambiente e atendimento ao familiar ${ }^{(13)}$. 0 desconhecimento dos familiares, quando questionados em tópico relativo à equipe multiprofissional, apontou para importância do trabalho da equipe em direção à família, para conhecerem a diversidade de profissões que atuam em Unidade de Terapia Intensiva.

Os momentos da visita na terapia intensiva são os mais importantes para os familiares, pois é um tempo para ficar mais próximo do parente hospitalizado, diminuindo, assim, o distanciamento da relação, ocasionado pelo adoecimento. No entanto, somente permitir a entrada da família no ambiente não é suficiente para proporcionar conforto, é necessário preparar e acompanhar a família durante a visita, para identificar e esclarecer as dúvidas ${ }^{(14)}$. Nesse tópico da cartilha, os familiares desejaram abordagem mais ampla para se sentiram mais seguros. Esse preparo perpassa pela apresentação da dinâmica da unidade, das circunstâncias que podem encontrar o parente, possivelmente com vários dispositivos, passa, ainda, por direcionamentos quanto a condutas da família ao lado do fami$\operatorname{liar}^{(15)}$. Por isso, o instrumento validado intencionou tocar em conteúdo que contemplasse os aspectos mais comuns dessa experiência, para instrumentalizar familiares.

De modo geral, o instrumento informativo " $\mathrm{O}$ que você precisa saber quando tem um parente na Unidade de Terapia Intensiva?" foi avaliado de forma positiva pelos juízes especialistas participantes. A análise do material subsidiou o aprimoramento da cartilha quanto às informações, à estrutura e apresentação. As colocações dos juízes apontaram efeito benéfico no uso de material informativo, dentro e fora do ambiente hospitalar. A obtenção das informações por meio da cartilha, em momentos com baixo nível de estresse, por exemplo em casa, e sempre que julgarem necessário, tem se mostrado eficaz, uma vez que obter informações diretamente dos profissionais em ambiente com fatores estressores pode comprometer a capacidade de apreensão das notícias e informes.

A avaliação semântica demonstrou que o conforto para famílias esteve associado ao fato de receber informações claras a respeito da dinâmica da unidade 
de internamento e, principalmente, sobre a evolução clínica do familiar. Além disso, a validade e a relevância do material elaborado residem na verificação de que as expectativas e prioridades dos familiares frente a essa experiência foi contemplado no conteúdo elaborado, apontado como suficiente e de fácil compreensão, portanto, apto a melhorar o conhecimento e a satisfação de familiares.

Recomenda-se o uso da cartilha informativa imediatamente após admissão do paciente na terapia intensiva, podendo ser oferecida por qualquer profissional de saúde, antes da primeira visita, para que o familiar possa realizar a leitura do material e ser beneficiado com elementos que o auxilie no enfrentamento do adoecimento de um membro do grupo familiar. Neste sentido, o uso das tecnologias educativas pode advir como suporte cuidativo-educativo, contribuindo para o acolhimento e a educação em saúde de familiares com pessoas internadas em unidade de terapia intensiva.

\section{Conclusão}

Os índices de validade de conteúdo apontaram que o instrumento elaborado contempla as informações de interesse dos familiares que vivenciam o processo de hospitalização, podendo ser material educativo útil para ajudá-los a enfrentar de modo mais fortalecido essa realidade, uma vez que a desinformação é um dos principais difusores de desconfortos.

\section{Colaborações}

Bezerra JS contribuiu com redação do artigo, projeto, análise e interpretação de dados. Freitas KS colaborou com concepção, projeto, análise e interpretação de dados e revisão crítica relevante do conteúdo intelectual. Góis JA e Lima AB auxiliaram na redação do artigo, análise, interpretação dos dados e aprovação final da versão a ser publicada. Fontoura EG e Oliveira MAN cooperaram com a redação do artigo.

\section{Referências}

1. Freitas KS, Mussi FC, Menezes IG. Discomfort experienced at the daily life of relatives of people admitted at ICU. Esc Anna Nery. 2012; 16(4):704-11. doi: dx.doi.org/10.1590/S141481452012000400009

2. Mendes AP. Impact of critical illness news on the family: hermeneutic phenomenological study. Rev Bras Enferm. 2018; 71(1):170-7. doi: http://dx. doi.org/10.1590/0034-7167-2016-0163

2. Oliveira CN, Nunes EDCA. Caring for family members in the ICU: challenges faced by nurses in the interpersonal praxis of user embracement. Texto Contexto Enferm. 2014; 23(4):954-63. doi: http:// dx.doi.org/10.1590/0104-07072014003590013

3. Valente CO Fonseca GM, Freitas KS, Mussi FC. Family comfort to a relative in the intensive therapy unit. Rev Baiana Enferrm. 2017; 31(2):e17597. doi: http://dx.doi.org/10.18471/rbe.v31i2.17597

4. Varela AIS, Rosa LM, Salum VRNC, Souza AIJ. Educational booklet for patients in palliative care and their families: construction strategies. Rev Enferm UFPE on line [Internet]. 2017 [cited Aug 13, 2019]; 11(Supl 7):2955-62. Available from: https://periodicos.ufpe.br/revistas/revistaenfermagem/article/view/11110/19210

5. Oliveira SC, Lopes MVO, Fernandes AFC. Development and validation of an educational booklet for healthy eating during pregnancy. Rev Latino-Am Enfermagem. 2014; 22(4):611-20. doi: http://dx. doi.org/10.1590/0104-1169.3313.2459

6. Ivo RS, Ribeiro LM, Leon CGRMP, Scardosim JM, Guarda LEDA, Beleza LO. Maternal perception and construction of an educational material on phototherapy. Rev Enferm UFPE on line [Internet]. 2017 [cited Aug 13, 2019]; 11(3):1207-15. Available from: https://periodicos.ufpe.br/revistas/revistaenfermagem/article/view/13496/16222

7. Nascimento MHM, Teixeira E. Educational technology to mediate care of the "kangaroo family" in the neonatal unit. Rev Bras Enferm. 2018; 71(Supl 3):1370-7. doi: http://dx.doi.org/10.1590/00347167-2017-0156

8. Berardinell LM, Guedes NA, Ramos JP, Silva MG. Educational technology as a strategy for the empowerment of people with chronic illnesses. Rev 
Enferm UERJ. 2014; 22(5):603-9. doi: https://doi. org/10.12957/reuerj.2014.15509

9. Freitas KS, Menezes IG, Mussi FC. Validation of the Comfort scale for relatives of people in critical states of health. Rev Latino-Am Enfermagem. 2015; 23(4):600-8. doi: http://dx.doi. org/10.1590/0104-1169.0180.2601

10. Coluci MZO, Alexandre NMC, Milani D. Content validity in the development and adaptation processes of measurement instruments. Cienc Saúde Coletiva. 2011; 16(7):3061-8. http://dx.doi. org/10.1590/S1413-81232011000800006

11. Othman H, Subramanian P, Mohd NA, Hassan H, Haque M. The effect of information booklets on family members' satisfaction with decision making in an intensive care unit of Malaysia. J Young Pharm. 2016; 8(2):128-32. doi: http://dx.doi. org/10.5530/jyp.2016.2.13

12. Vasconcelos EV, Freitas KO, Torres, RS, Silva SED, Baia RSN, Araújo JS, et al. The importance of com- munication: family members of patients at an intensive care unit. Rev Conexão. 2016; 12(2):196206. doi: http://dx.doi.org/10.5212/Rev.Conexao.v.12.i2.0002

13. Passos SSS, Silva JO, Santana VS, Santos VMN, Pereira A, Santos LM. User embracement in care for families at an intensive care unit. Rev Enferm UERJ. 2015; 23(3):368-74. doi: http://dx.doi. org/10.12957/reuerj.2015.6259

14. Davidson JE, Aslakson RA, Long AC, Puntillo KA, Kross EK, Hart J, et al. Guidelines for familycentered care in the Neonatal, Pediatric, and Adult ICU.CritCare Med.2017; 45(1):103-28. doi: http:// dx.doi.org/10.1097/CCM.0000000000002169

15. Gibaut MAM, Hori LMR, Freitas KS, Mussi FC. Comfort of the patient's family in an intensive care unit related to welcoming. Rev Esc Enferm USP. 2014; 47(5):117-24. doi: http://dx.doi.org/10.1590/ S0080-623420130000500015 